\title{
Volume Perception in the Cyclic Display of One Stroke Apparent Motion and ISI (Inter Stimulus Interval)
}

\author{
Xiahong Cheng, Masanori Idesawa \\ The University of Electro-Communications, Tokyo, Japan
}

\begin{abstract}
3D feature perception from motion has been studied as " $X$ ' from motion". As for the "X", "depth", "shape", "structure", and "surface" have been reported widely; recently, "volume" was added as a new category. It was shown that ADPs (appearing and disappearing parts) plays an important role for the volume perception. In the present study, we examined these perception in the velocity field produced by the cyclic display of one stroke apparent motion sequence and suitable ISI (inter stimulus interval); in the observation experiment of moving random dot pattern stuck on different types of surface, it was found that not only the "depth", "structure", "shape", and "surface", but also the "volume" can be perceived almost the same as in the real continuous motion. By using the random dot pattern stuck on the cylindrical objects with and without ADPs were presented in almost the same situation; then, it was proved that the ADPs plays one of the important factors for the volume perception in the cyclic display of one stroke apparent motion and ISI.
\end{abstract}

Keywords: X from motion, apparent motion, cyclic display, depth, structure, surface, volume

\section{Introduction}

Human vision has ability to perceive 3D features from motion, which has been studied as " $\mathrm{X}$ ' from motion" (Ullman, 1984; Cornilleau-Pérès \& Droulez, 1994). As for the "X", "depth", "shape", "structure", and "surface" have been reported widely; recently, as a new category "volume" was added (Cheng, Idesawa, \& Wang, 2010; Cheng \& Idesawa, 2011). In the previous study, we performed the observation experiment of moving random dot pattern stuck on different types of surface; then it was confirmed that "depth", "shape", "structure", "surface", and also the "volume" were perceived successfully (Cheng et al., 2010; Cheng \& Idesawa, 2011). In addition, it was proved that the ADPs (appearing and disappearing parts) plays one of the important factors for the volume perception.

In the present study, we will examine these perception in the velocity field produced by the cyclic display of one stroke apparent motion sequence and suitable ISI (inter stimulus interval) (Mather, 2006; Idesawa, Chen, \& Wang, 2006; Idesawa, 2010); then, we will investigate the importance of ADPs for the the "volume" perception.

Volume perception is an important function in human visual system, which is the perception of a partially enclosed space filled with some medium. In the case of objects having a curved surface, such as cylinders and spheres, even when only the front surface of the object is visible, it can be perceived as that it

Xiahong Cheng, Master, UEC Museum of Communications, The University of Electro-Communications.

Masanori Idesawa, Ph.D., Professor Emeritus, UEC Museum of Communications, The University of Electro-Communications. 
has volume, forming a partially enclosed space filled with some medium, as a result, the object is recognized as a solid.

The first reports on the volume perception pertained to the perception of an illusory solid object from binocular viewing (Idesawa, 1991a; 1991b; 1993). Perception of not only an illusory opaque solid object but also an illusory transparent object with volume has been attributed to a transparent illusory surface perception and a pantomime effect in binocular viewing (Zhang, Idesawa, \& Sakaguchi, 1998; Idesawa \& Zhang, 1999), in which a partially enclosed space filled with a transparent medium was perceived.

In the further study of volume perception in binocular viewing of real and simulated cylindrical objects, it was confirmed that BURs (binocularly unpaired regions on an object) play an important role for the volume perception (Iwamoto \& Idesawa, 1997; Watanabe \& Idesawa, 2001). And in the recent study of volume perception from motion, successive ADPs were found to be an important factor for volume perception of moving objects (Cheng et al., 2010; Cheng \& Idesawa, 2011), which is considered to be compatible with binocular unpaired parts in stereopsis.

In the present paper, we will study volume perception from the velocity field produced by the cyclic display of one stroke apparent motion pairs with random dots images (Mather, 2006; Idesawa et al., 2006; Idesawa, 2010). With which, the objects with and without ADPs could be presented in almost the same situation; then, conduct the experiment to investigate and discuss the importance of ADPs for the volume perception in the cyclic display of one stroke apparent motion and suitable ISI.

\section{Volume Perception From Motion}

We performed the observation experiment of moving random dot pattern stuck on different types of surface; then it was confirmed that "depth", "shape", "structure", "surface", and "volume" were perceived successfully from continuous real motion.

In the former works, we studied the relation between volume perception from motion and the ADPs (Cheng et al., 2010; Cheng \& Idesawa, 2011). By using computer models, the psychophysical experiments were designed and performed to simulate the cylindrical object moving in the 3D model space. In computer models, random dots stuck on the object's surface were used to present the objects, and then projected onto the screen to generate the stimuli that were shown to the subjects.

Experiments were performed for three basic motion patterns (rotation, rolling, and translation) and for six moving orientations $(0, \pi / 6, \pi / 3, \pi / 2,2 \pi / 3$, and $5 \pi / 6)$ (see Figure $3(\mathrm{a})-(\mathrm{d})$ ). In all conditions (that is, combination of motion pattern and moving orientation), volume perception could be obtained remarkably more easily from the stimuli with ADPs than from the stimuli without ADPs (Figure3 (e)) (Cheng et al., 2010; Cheng \& Idesawa, 2011).

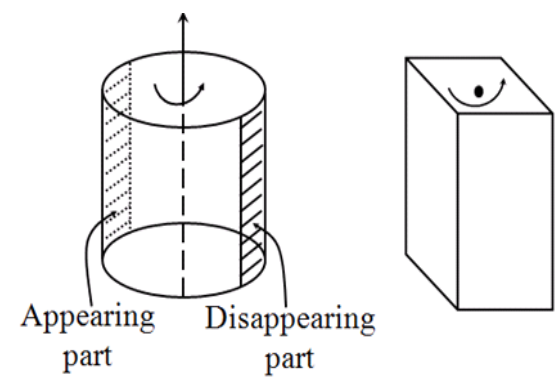

Figure 2. ADPs in rotating cylindrical and polyhedral object. 


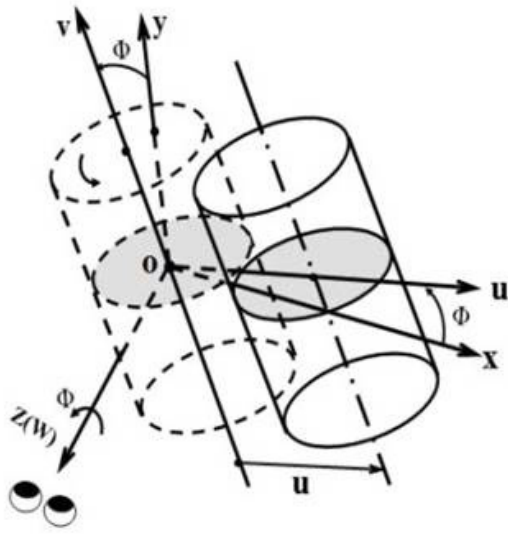

(a)

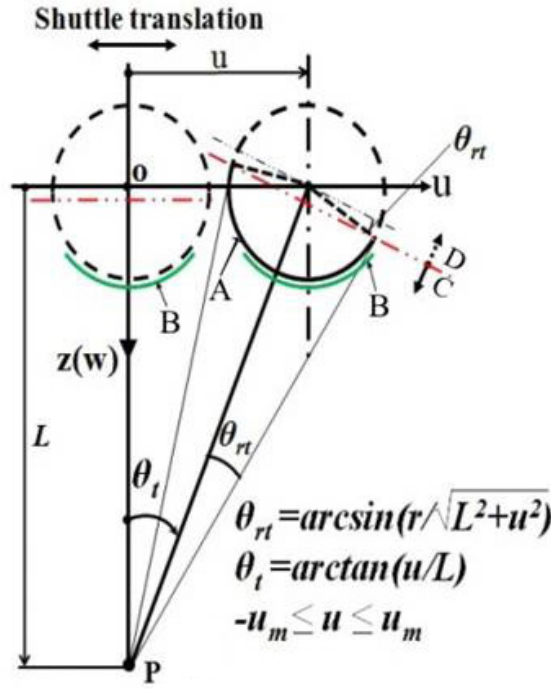

(c)

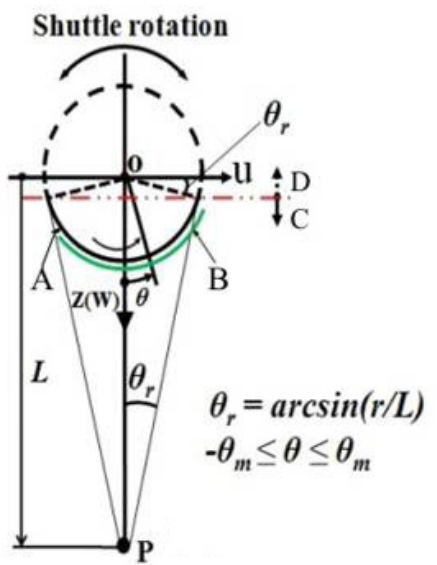

(b)

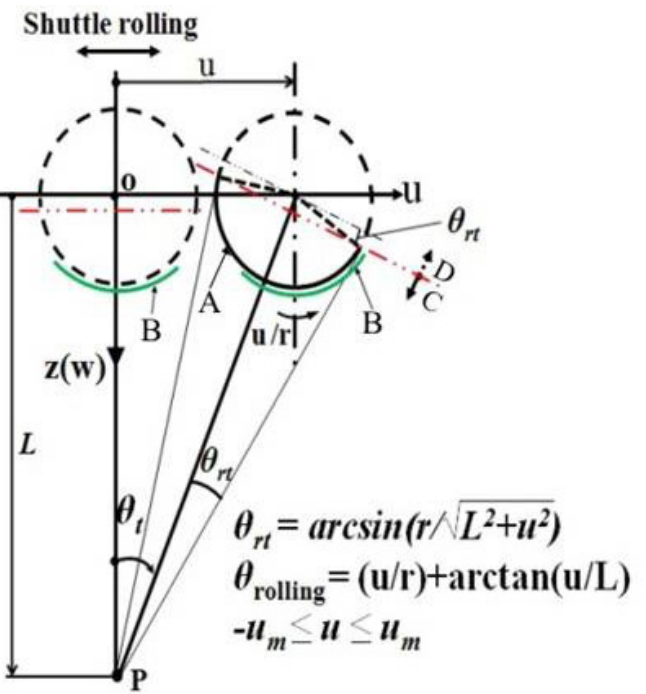

(d)

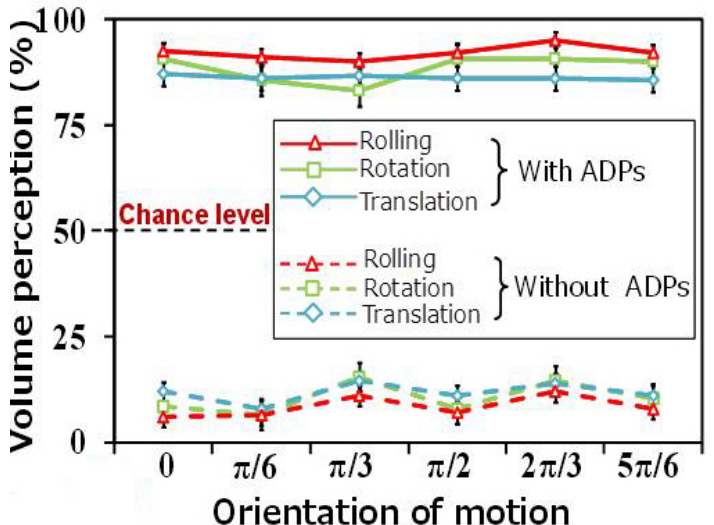

(e)

Figure 3. Three types of shuttle motion (rotation, translation, and rolling) with and without ADPs and volume perception ((a) Configuration of the cylindrical object in both the kinematic and space coordinate systems; (b) Configuration of shuttle rotational motion; (c) Configuration of shuttle translational motion; (d) Configuration of shuttle rolling; and (e) Experimental results of volume perception rates for three types of shuttle motion in different orientations $(0, \pi / 6, \pi / 3, \pi / 2,2 \pi / 3$, and $5 \pi / 6)$. Average volume perception rates for 10 subjects are shown; error bars show the deviation between subjects (Cheng et al., 2010)). 
Furthermore, beside the cylindrical object model in which the ADPs are caused by the self-occlusion, the partially visible cylindrical object model and polyhedral object model were also examined (Cheng \& Idesawa, 2011). It is corresponding to the situation that the moving object is partially sinking into some liquid and the sinking parts were occluded by the liquid surface. Similar to the result gotten from the experiments of cylindrical object model, it was found that the volume perception could be obtained for both partial cylindrical object model and polyhedral object model with ADPs which were not generated by self-occlusion, but could hardly be obtained for the stimuli without ADPs. And in such object models, the obtaining of volume perception was not affected by the kind of motion patterns or the moving orientation. It should be noted here that the polyhedral object model which was difficult to be perceived as volumetric in the former observation (Cheng et al., 2010), with the ADPs produced by occlusion with proximal surfaces, could also be obtained volume perception from motion (Cheng \& Idesawa, 2011).

\section{Velocity Field Produced by the Cyclic Display of One Stroke Apparent Motion and Suitable ISI}

Apparent motion, different from the continuous motion in the real world, is the motion that generated from rapidly alternating a succession of the still images (which are slight different from each other). Mather (2006) displayed a new illusion of unidirectional motion perception from cyclic display of two sequential images and a blank frame (ISI): two sequential frames causing apparent motion and a blank image suppressing the apparent motion in opposite direction. This phenomenon can be produced by using three phase frames including two correlated frames which can produce apparent motion and the one independent image such as blank image (ISI) (see Figure 4(a)). We used the random dots instead of the images to remove the pictorial depth cue from Mather's case. By using two phase-images with random dots (the dots in each phase-image have some relation with the other) and one independent image such as blank, they succeeded in generating unidirectional motion perception which we call as the velocity field from the cyclic display of one stroke apparent motion with suitable ISI (Idesawa et al., 2006; Idesawa, 2010).

As shown in Figure 4(b), we tried to produce the various kinds of velocity field distribution with the velocity field by the cyclic display of the three phase images including correlated image pair (Ia, Ib) which produces one stroke apparent motion and independent image (Ic); the velocity field is formed and optic flow can be perceived as a stream along the apparent motion stroke which was resulted from the difference between the correlated image pair.

We designed the correlated image pair with random dots and successfully displayed various types of the continuous velocity field distribution (see Figure 4(c)) (Idesawa et al., 2006). For instance, from the distribution of velocity field shown in Figure 4(c), we can perceive 3D swelling surface as shown in Figure 4(d). In addition, the direction (polarity) of the velocity field is reversed by changing the cyclic order of the correlated image pair. 

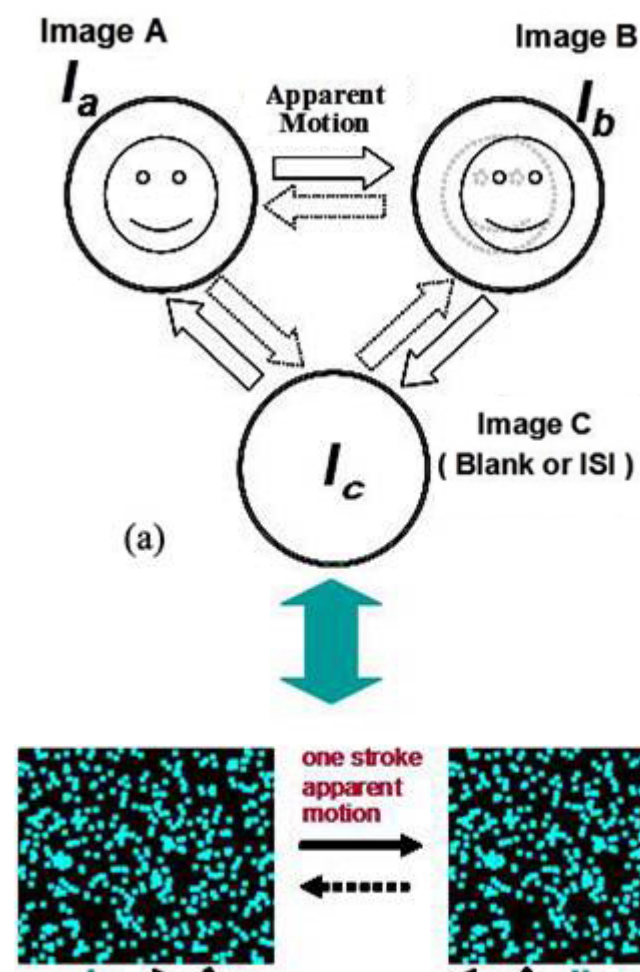

la

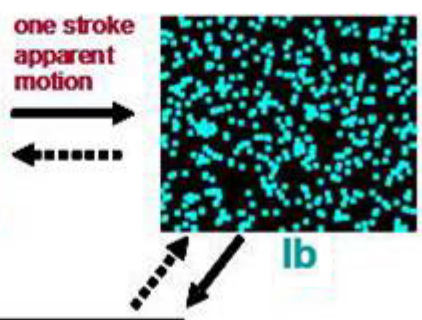

(c)

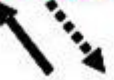

Ic

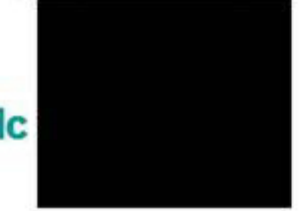

(b)
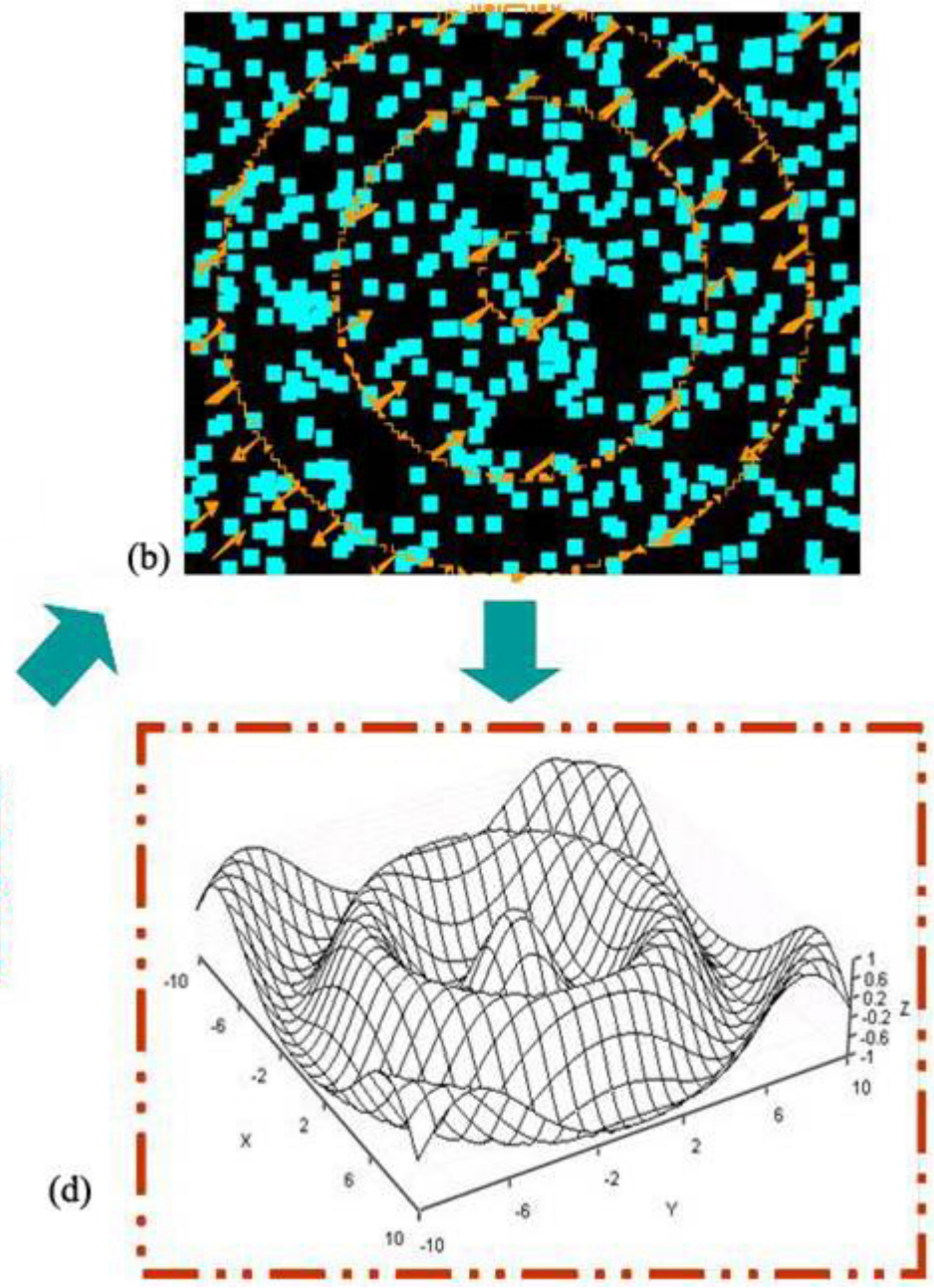

(d)

Figure 4. Examples of 3D swelling surface perception from the velocity field produced by cyclic display of one stroke apparet motion with ISI ((a) Conceptual figure showing the three phase image presentation; (b) Conceptual figure showing the cyclic display of one stroke apparent motion and ISI of blank image; (c) Conceptual figure showing the velocity field produced in (b); and (d) Conceptual figure showing 3 D surface perceived) (Idesawa et al., 2006).

\section{Volume Perception From the Velocity Field Produced by Cyclic Display of One Stroke Apparent Motion and ISI}

Not only the 3D surfaces but also the volumetric objects can be perceived from the velocity field produced by the cyclic display of one stroke apparent motion and suitable ISI almost the same as that in the real motion. Volume perception in apparent motion was tried and proved that the ADPs play an important factor for the volume perception from velocity field as shown in Figure 5.

We observed the rotational apparent motion in various directions with the repetition of one stroke apparent motion and ISI; then, we found that almost the same perceptions as in real continuous motion were obtained. In the present study, we examined only for the motion in horizontal direction, which is corresponding motion orientation of 0 degree in Figure 3(a), by the method of three alternative forced choices (volume/ not sure/ curved surface); then the perceptual results shown in Figure 5 was obtained. Meaningful volume perception rate could be obtained for with ADPs, but could not be for without the ADPs. 
The experiment was conducted in a dark room. The monocular images used to simulate objects with and without ADPs in apparent motion were shown on a 19-inch CRT screen $(1280 \times 1024$ pixel, $120 \mathrm{~Hz}$ frame rate). The computer used in the experiment had a CPU of Intel (R) Pentium (R) 4 with $3.4 \mathrm{G} \mathrm{Hz}$, and the OS was Windows XP version. The observation distance was $60 \mathrm{~cm}$, and the screen size was $40.5 \times 30 \mathrm{~cm}^{2}$.

In the experiment, subjects observed the test stimuli with both eyes open and with free observation. The subjects indicated what they perceived in three options: (1) cylindrical object with volume; (2) just a curved surface; and (3) not sure. The stimuli simulating cylindrical objects with and without ADPs in each motion pattern were displayed 20 times, and totally all stimuli were displayed 120 times in a random order. The response of the subject was recorded and then the next stimulus was displayed. This procedure was repeated until the entire set of stimuli had been displayed and all responses were accumulated for all subjects. Three subjects were attended in this experiment and the results are shown in Figure 5. Especially in the rotation and rolling motion pattern, it was found that most of the subjects can get the volume perception of the object when the stimulus had ADPs; while in the case of the stimulus without ADPs, most of subjects could only get the perception of a curved surface. That is, it was proved that ADPs plays an important role for volume perception from the velocity field in the case of cyclic display of one stroke apparent motion and ISI.

(a)
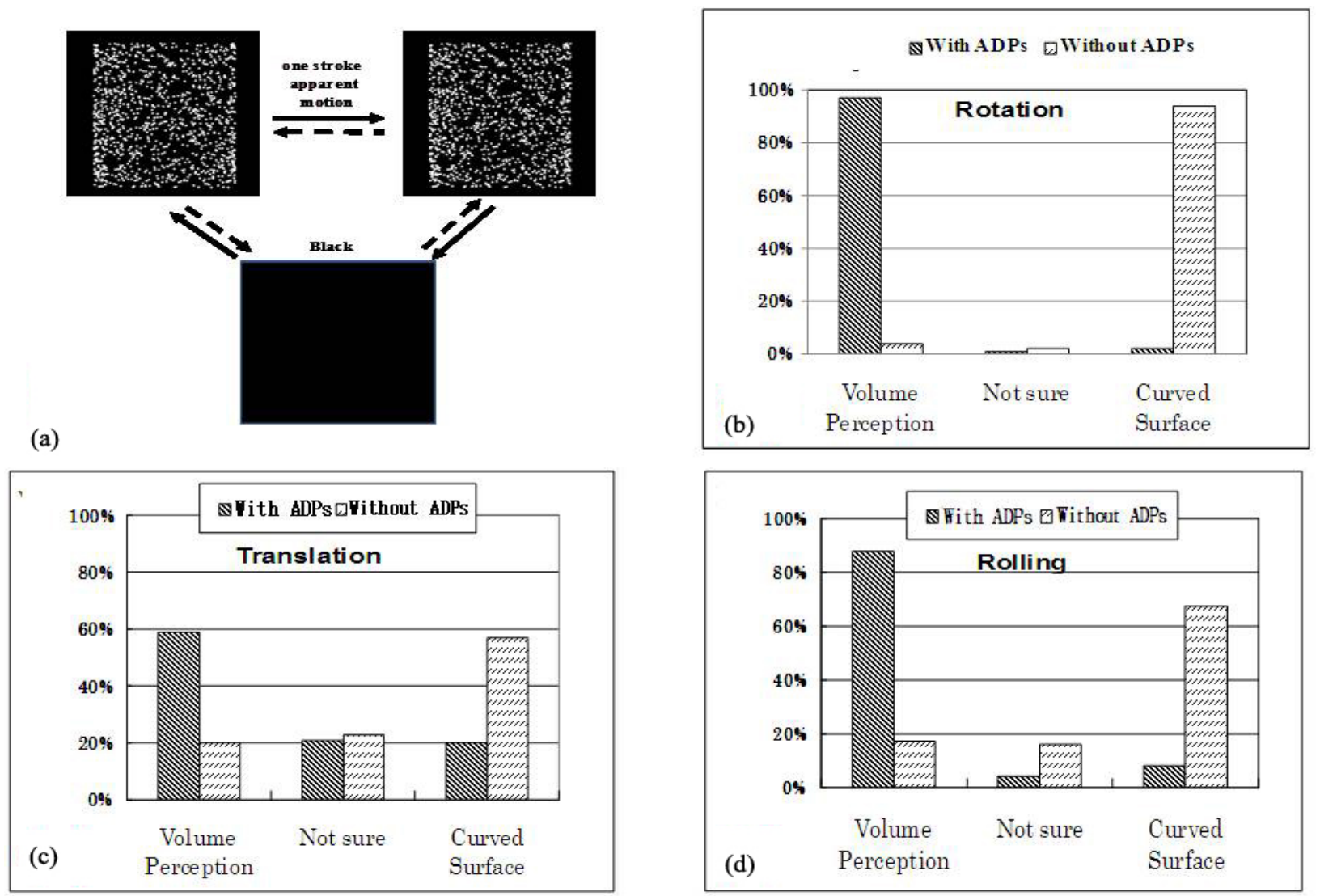

Figure 5. Volume perception from velocity field by cyclic display of one stroke apparent motion with ISI ((a) An example of cyclic display of the 3-phase images producing the velocity field of random dots stuck on cylindrical surface; (b) Volume perception rates for three options in apparent rotation; (c) Volume perception rates for three options in apparent translation; and (d) Volume perception rates for three options in apparent rolling). 


\section{Discussions and Conclusion}

In this paper, we observed the random dot pattern stuck on different types of surface for the cyclic display of one stroke apparent motion with ISI; then it was confirmed that "depth", "shape", "structure", "surface", and "volume" could be perceived successfully. Volume perception in the cyclic display of one stroke apparent motion and ISI was studied for with and without ADPs; then it was found that volume perception could be obtained easily for with ADPs but difficult to be obtained for without ADPs. Based on the result, it was inferred that ADPs bring volume perception in the cyclic display of one stroke apparent motion and ISI.

In both of the case of shuttle motion (Figure 3(e)) and the case of velocity field (Figure 5), percentage of volume perception for translation motion was remarkably lower than these in rotation and rolling. It could be interpreted that when the stimulus with ADPs moved in the motion pattern of rotation and rolling, the percentages of volume perception were remarkably higher than that of in the motion pattern of translation. As for the reason of such result, the difference of the size of the ADPs in the three motion patterns could be thought as one main reason. In cylindrical object rotating around its' axis, the ADPs were the surfaces corresponding to the shift angle $\theta$ (Figure 3). In the case of translation, the cylindrical object moved along the horizontal axis and the ADPs only depend on the horizontal displacement that lead to the change of the visible part; then, the ADPs in translation was smaller than that of in the rotation and rolling. Accordingly, the volume perception of cylindrical objects in translation was weaker than the case in both the case of rotation and rolling. In addition, in the case of continuous shuttle motion (Figure 3(e)), rolling motion mode gives the most significant features of volume perception; while in the case of apparent motion (Figure 5(d)), rolling gives less significant features of volume perception than rotation mode. It could be interpreted that the rolling motion was more complicated motion pattern than that of rotation, then the one stroke motion with only the two terminal pictures were insufficient to represent rolling motion faithfully. We observed the multi-stroke apparent motion with intermediate pictures between two terminal pictures, then it was confirmed that the rolling motion pattern with the two stroke apparent motion gives more significant volume perception features than that of with the one stroke apparent motion.

\section{References}

Cheng, X., \& Idesawa, M. (2011). With occlusion by proximal surfaces, even without self-occlusion, continuously appearing and disappearing parts bring volume perception from motion. Optical Review, 18(4), 297-300.

Cheng, X., Idesawa, M., \& Wang, Q. (2010). Volume perception from motion. Optical Review, 17(5), 439-442.

Cornilleau-Pérès, V., \& Droulez, J. (1994). The visual perception of three-dimensional shape from self-motion and object motion (pp. 2331-2336). Great Britain: Pergamon (Eisevier Science Ltd.).

Idesawa, M. (1991a). Perception of 3-D illusory surface with binocular viewing. Japanese Journal of Applied Physics, 30, $751-754$.

Idesawa, M. (1991b). Perception of 3-D transparent illusory surface in binocular fusion. Journal of Applied Physics, 30, 1289-1292.

Idesawa, M. (1993). Two types of occlusion cues for the perception of 3-D illusory objects in binocular fusion. Journal of Applied Physics, 32, L75-L78.

Idesawa, M. (1997). A study on visual mechanism with optical illusions. Journal of Robotics and Mechatronics, 9(2), 85-91.

Idesawa, M., \& Zhang, Q. (1999). Newly found visual illusion and 3-D display (IEICE Trans.). Electron, 82(C), 1823-1830.

Idesawa, M., Chen, X., \& Wang, Q. (2006). A new type of visual effect produced by cyclic display of a pair images with slight disparity. Proceedings of IDW'06, 2321-2324.

Iwamoto, T., \& Idesawa, M. (1997). Volume perception and a processing method of unpaired region in stereo vision. J. Robotics Mechatronics, 9(2), 121-125. 
Mather, G. (2006). Two-stroke: A new illusion of visual motion based on the time course of neural responses in human visual system. Vision Research, 46, 2015-2018.

Watanabe, O., \& Idesawa, M. (2001). Effect of an occluding contour on the volume perception in binocular stereopsis. Japanese Journal of Applied Physics, 40, 958-960.

Ullman, S. (1984). Maximizing rigidity: The incremental recovery of 3-D structure from rigid and rubbery motion. Perception, 13, 255-274.

Zhang, Q., Idesawa, M., \& Sakaguchi, Y. (1998). Pantomime effect in the perception of volumetrical transparent illusory object with binocular viewing. Japanese Journal of Applied Physics, 37(3B), L329-L332. 